\title{
PROFISSIONAIS DE SAÚDE, CUIDADOS PALIATIVOS E FAMÍLIA: REVISÃO BIBLIOGRÁFICA
}

\author{
Aline Camilo Lima ${ }^{1}$, José Augusto de Souza Silva², Maria Julia Paes da Silva ${ }^{3}$
}

\begin{abstract}
RESUMO: Objetivo: identificar e analisar através de revisão bibliográfica sistematizada a comunicação entre o profissional de saúde e a família de pacientes em fase terminal, e como é abordada a dimensão família nos Cuidados Paliativos. Método: pesquisa quantitativa exploratória, baseada no material indexado nas bases de dados Dedalus, Lilacs, MedLine, PeriEnf e Scielo, entre 1997 a 2008, utilizando-se como descritores: Cuidados Paliativos, Comunicação e Família. Resultados: foram identificados 127 artigos com os descritores Comunicação e Cuidados Paliativos e 34 com Comunicação, Cuidados Paliativos e Família. A MedLine foi a base de dados que apresentou maior número de artigos (22) para os descritores Comunicação, Cuidados Paliativos e Família, sendo os EUA o país com mais publicações (14) para esses descritores. Conclusões: O número de publicações que considera a família como foco do cuidado paliativo é pequeno. É através da comunicação que o profissional se aproxima da família.
\end{abstract}

PALAVRAS-CHAVE: Comunicação; Cuidados paliativos; Família.

\section{HEALTH PROFESSIONALS, PALLIATIVE CARE AND FAMILY: A BIBLIOGRAPHIC REVIEW}

\begin{abstract}
Objective: to identify and analyze through systematic literature review the issue of communication between the health professional and family of patients in the terminal stage, and how family is addressed in Palliative Care. Method: exploratory and quantitative research, based on material indexed in the databases Dedalus, Lilacs, MedLine, PeriEnf and Scielo, from 1997 to 2008, using descriptors such as "Palliative Care”, “Communication” and "Family”. Results: 127 articles were identified by the descriptors "Communication and Palliative Care", and 34 by the descriptors "Communication, Palliative Care and Family”. The MedLine database was the one that showed the largest number of articles (22) with the descriptors "Communication, Palliative Care and Family", and the U.S. was the country with more publications (14) with these descriptors. Conclusions: The number of publications that considers the family as a focus of palliative care is small. It is through communication that the professional approaches the family.
\end{abstract}

KEYWORDS: Communication; Palliative care; Family.

\section{PROFESIONALES DE LA SALUD, LOS CUIDADOS PALIATIVOS Y FAMILIA: REVISÍON BIBLIOGRÁFICA}

RESUMEN: Objetivo: identificar y analizar a través de revisión bibliográfica sistematizada la comunicación entre el profesional de la salud y la familia de pacientes en fase terminal, y cómo es abordada la dimensión familia en los Cuidados Paliativos. Método: investigación cuantitativa exploratoria, basada en el material indexado en las bases de datos Dedalus, Lilacs, MedLine, PeriEnf y Scielo, entre 1997 a 2008, utilizándose como descriptores Cuidados Paliativos, Comunicación y Familia. Resultados: fueron identificados 127 artículos con los descriptores Comunicación y Cuidados Paliativos y 34 con Comunicación, Cuidados Paliativos y Familia. La MedLine fue la base de datos que presentó mayor número de artículos (22) para los descriptores Comunicación, Cuidados Paliativos y Familia, siendo los EE.UU el país con más publicaciones (14) de estos descriptores. Conclusiones: El número de publicaciones que considera la familia como foco del cuidado paliativo es pequeño. Es a través de la comunicación que el profesional se acerca a la familia.

PALABRAS CLAVE: Comunicación; Cuidados paliativos; Familia.

${ }^{1}$ Graduanda em Enfermagem pela Escola de Enfermagem da Universidade de São Paulo-USP.

${ }^{2}$ Graduando em Enfermagem pela Escola de Enfermagem da USP.

${ }^{3}$ Doutora. Professora Titular do Departamento de Enfermagem Médico-Cirúrgica da Escola de Enfermagem da USP.

Autor correspondente:

Maria Julia Paes da Silva

Av. Dr. Eneas de Carvalho Aguiar, 419 - 05403-000 - São Paulo-SP

Recebido: 09/11/08

E-mail: maria.paes@pq.cnpq.br

Aprovado: 20/06/09

Cogitare Enferm 2009 Abr/Jun; 14(2):360-7 


\section{INTRODUÇÃO}

O processo de doença cria uma expectativa para aqueles que cuidam: a cura. Para estudantes e profissionais da área de saúde, curar significa vitória, pois a resposta de um caso enigmático foi descoberta, a última peça do "quebra-cabeça" foi colocada, apresentando àqueles que participaram da montagem uma bela paisagem. Terminar um "quebra-cabeça", assim como conseguir alcançar a cura de um paciente realmente traz uma sensação de bem-estar. Mas o que sentimos quando não encontramos a última peça, quando não alcançamos a cura? Sentimos desespero, raiva e muitas vezes acabamos desistindo: Não há mais nada para fazer.

Essa última frase deve soar familiar para alguns. Infelizmente é a fala que muitos pacientes fora das possibilidades terapêuticas de cura ouvem quando o tratamento ao qual estão submetidos não atinge o objetivo principal que eles e os profissionais tanto esperam. A situação complica-se ainda mais porque além do paciente, os profissionais de saúde devem encontrar algum jeito de informar à família o que está acontecendo.

Uma das justificativas colocadas para tentar responder o porquê da dificuldade que as pessoas têm para trabalhar com pacientes em fase terminal e sua família, é a falta ou pouca abordagem do tema nos cursos da área da saúde. Outras justificativas, como: interesse dos alunos pelo assunto, falta de paciência para lidar com famílias neste âmbito ou até mesmo dificuldade em relação à morte, também são relevantes ${ }^{(1)}$.

O fato é que nem sempre a cura será o objetivo principal dos profissionais de saúde; o que os pacientes em fase terminal e sua família almejam é uma qualidade de vida nesses últimos momentos, uma atenção voltada ao cuidado integral, que tem o paciente como um ser holístico. Muitas vezes, a família não só tem que participar, mas espera poder ser parte integrante do tratamento; esse quadro nos remete à essência dos Cuidados Paliativos.

Os hospices, lugares conduzidos por religiosos, eram assim designados ao cuidado de viajantes, peregrinos, doentes esquecidos pelo governo e outros que necessitavam de ações humanitárias e que não poderiam pagar por elas ${ }^{(2-3)}$. Com a evolução e 0 predomínio do modelo curativista os profissionais desistiram desse tipo de lugar e transferiram-se para instituições hospitalares cujo objetivo era cuidar de pessoas que ainda tinham chances de cura.

Mesmo assim, os hospices continuaram com o seu objetivo de prover apoio e cuidados espirituais e físicos tanto quanto fossem possíveis. Foi neste contexto que, em 1950, Cicely Saunders fundou o St. Christopher Hospice em Londres, com a ideia de afirmar o cuidado daqueles que se aproximavam da morte ${ }^{(2)}$. A fundadora era formada em Enfermagem, Serviço social e Medicina; ninguém melhor do que uma profissional da saúde para preocupar-se com o descaso social em que viviam os pacientes em fase terminal. Essa preocupação trouxe consigo a criação da filosofia dos Cuidados Paliativos-CP, que em suma é cuidar das pessoas com a intenção de aliviar o sofrimento e ajudá-las a encontrar e alcançar uma melhor qualidade de vida, mesmo que a doença esteja em um estágio avançado, ativo e progressivo.

Em 1990, a Organização Mundial da SaúdeOMS definiu CP como:

Cuidado ativo e total dos pacientes cuja enfermidade não responde mais aos tratamentos curativos. O objetivo dos Cuidados Paliativos é atingir a melhor qualidade de vida possível para os pacientes e sua família ${ }^{(4)}$.

Este conceito foi redefinido em 2002 pela própria OMS, porém com ênfase no alívio do sofrimento:

Cuidados Paliativos é uma abordagem que aprimora a qualidade de vida dos pacientes e famílias que enfrentam problemas associados com doenças ameaçadoras de vida, através da prevenção e alívio do sofrimento, por meio de identificação precoce, avaliação correta e tratamento da dor e outros problemas de ordem física, psicossocial e espiritual ${ }^{(4)}$.

A OMS ${ }^{(4)}$ também esclarece que a filosofia dos Cuidados Paliativos afirma a vida e trata a morte como um processo normal, não apressa nem adia a morte, integra os aspectos psicossociais e espirituais nos cuidados do paciente, oferece um sistema de apoio e ajuda aos pacientes para viver tão ativamente quanto possível até a morte, disponibiliza um sistema de apoio para auxiliar o paciente e sua família a lidar com a situação durante a doença do paciente e no processo de luto, exige uma abordagem em equipe e afirma que esses cuidados são aplicáveis no estágio inicial da doença, concomitantes com as modificações da doença e terapias que prolongam a vida.

No Brasil, o Sistema Único de Saúde-SUS, através de algumas ações, vem estimulando as práticas de Cuidados Paliativos como, por exemplo, o 
fortalecimento da política de implantação dos Cuidados Paliativos em todos os serviços de assistência especializada em HIV/aids, melhorando assim, a qualidade de vida dos usuários desses serviços ${ }^{(5)}$.

Perante a definição e filosofia dos Cuidados Paliativos, percebemos que a família é colocada também como foco da atenção. Mas, como ocorre a sua inclusão neste tipo de cuidado, qual o papel que lhe é designado e sua real importância mediante o processo de morrer de um de seus membros precisa ser periodicamente revista e recuperada.

Muitos autores já tentaram definir o que efetivamente é a família ou quem deve ser considerado como família sob o ponto de vista terapêutico. Talvez a melhor definição, ou a mais aceita atualmente, é a de que "a família é quem seus membros dizem que são"(6).

Para entendermos melhor a família no contexto de doença e morte, podemos recorrer ao modelo do móbile, proposto por Allmond, Buckman e Gofman. A família é como se fosse um móbile e cada membro é representado por uma peça do brinquedo. A soma das peças forma o todo e, por sua vez, todas elas, por mais diferentes que sejam, são feitas para ocuparem um lugar cujo resultado comum é o equilíbrio. A doença e a morte trabalham como eventos que desequilibram o sistema familiar, assim como se alguém removesse uma peça do móbile(6).

Tomando essa definição como norteadora de nosso trabalho, podemos verificar que a comunicação com o paciente e com os membros da família torna-se essencial para que os profissionais de saúde possam oferecer um serviço de qualidade, pois somente através de uma comunicação efetiva com todos os membros é que ele estará apto a incluí-la adequadamente na terapêutica dos cuidados paliativos, proporcionando neste novo contexto, outra forma de equilíbrio para ela ${ }^{(7)}$.

Apesar do grande avanço que os Cuidados Paliativos alcançaram nas últimas décadas, ainda assim podemos perceber que a família muitas vezes acaba adquirindo uma postura passiva diante do processo de morrer de um de seus membros, seja pelas rígidas normas hospitalares ou por uma visão arcaica de que somente a equipe de saúde pode tomar decisões sobre condutas terapêuticas ${ }^{(2)}$.

Muitos procedimentos acabam sendo impostos aos pacientes e seus familiares como a única alternativa de conduta. Desse modo, tanto a família como o paciente sentem-se ainda mais fragilizados e desamparados ao perceberem que suas opiniões, crenças, autonomia e valores não estão sendo respeitados, ocorrendo então o desgaste da relação de confiança entre os familiares, pacientes e equipe de saúde, gerando um distanciamento prejudicial à própria conduta terapêutica, deturpando a qualidade de vida de toda a família e se afastando das diretrizes dos Cuidados Paliativos que presam, justamente, pela inclusão da família no tratamento e a manutenção de sua qualidade de vida ${ }^{(8)}$. Entretanto, estudos mostram que, em cuidados paliativos domiciliares, a parceria entre profissionais de saúde e família é fundamental para que a terapêutica seja bem sucedida ${ }^{(9)}$.

Com tudo isso, faz-se necessário o estudo e a reflexão sobre como a família está sendo incluída nos cuidados paliativos. Não encontramos um estudo que abordasse a família em cuidados paliativos com este foco específico, portanto consideramos adequado fazer um levantamento sobre o tema, até para propor pesquisas futuras.

Esse trabalho permite verificar o quanto efetivamente tem-se pesquisado sobre a inclusão da família em cuidados paliativos, nos diferentes contextos de cuidado, explorando assim os benefícios dessa relação entre profissionais de saúde, paciente e família.

O presente estudo tem como objetivo identificar e analisar através de revisão bibliográfica sistematizada a comunicação entre o profissional de saúde e a família de pacientes em fase terminal e como é abordada a dimensão família nos Cuidados Paliativos.

\section{MÉTODO}

Trata-se de uma pesquisa quantitativa exploratória, baseada no material indexado nas bases de dados on-line Dedalus, Lilacs, MedLine, PeriEnf e Scielo, no período de 1997 a 2008. Foram incluídos artigos de periódicos nos seguintes idiomas: português, inglês e espanhol.

Os descritores utilizados na realização das buscas foram cuidados paliativos, comunicação e família, sendo permutados da seguinte forma: comunicação e cuidados paliativos; comunicação, cuidados paliativos e família. O uso dos descritores comunicação e cuidados paliativos foi necessário para que não se deixasse de lado nenhum artigo que envolvesse a família, mesmo que este termo não estivesse indexado como descritor. É importante ressaltar que, além da inclusão do maior número de artigos possíveis relacionados ao tema desse estudo, entendemos que a inclusão da família nos Cuidados 
Paliativos vem sendo analisada com foco na comunicação.

Os dados foram coletados e organizados de acordo com um instrumento que continha a base de dados onde o artigo foi encontrado, descritores utilizados, nome do artigo, autores, periódico, país, ano, objetivo do artigo, área (enfermagem, medicina, bioética, fisioterapia, serviço social, psicologia, interdisciplinar*, política ${ }^{\dagger}$ ), subárea (saúde do adulto e idoso, da criança, da mulher, saúde mental, saúde coletiva, diretrizes, ensino) e como aparece a dimensão "família" no artigo e resultado encontrado.

A análise dos dados foi feita a partir da leitura dos artigos e dos seus resumos (quando estes não foram encontrados) e posterior preenchimento das categorias citadas.

\section{RESULTADOS}

Foram identificados 127 artigos $(n=127)$ quando são utilizados os descritores comunicação $e$ cuidados paliativos, o $n$ total encontrado. Quando a busca é realizada utilizando-se como descritores comunicação, cuidados paliativos e família, este n diminui para 34; e através da análise dos dados, percebemos que os artigos que compõem este resultado $(n=34)$ estão inclusos nos artigos identificados com o uso dos descritores comunicação e cuidados paliativos.

O maior numero de artigos encontra-se na base de dados do MedLine, seguida do Lilacs e Dedalus.

Quadro 1- Identificação das bases de dados com abordagem do tema (n=127)

\begin{tabular}{|lccc|}
\hline Base de Dados & $\begin{array}{c}\text { Comunicação } \\
\text { e CP }\end{array}$ & $\begin{array}{c}\text { Comunicação, } \\
\text { CP e Familia }\end{array}$ & Total \\
MedLine & 101 & 22 & 123 \\
Lilacs & 15 & 8 & 23 \\
Dedalus & 7 & 4 & 11 \\
Scielo & 3 & 0 & 3 \\
Perienf & 1 & 0 & 1 \\
Total & 127 & 34 & 161 \\
\hline
\end{tabular}

O país responsável pela maior produção de artigos foi os Estados Unidos (73), seguido de Brasil

*Equipe Multiprofissional;

${ }^{\dagger}$ Programas e estratégias públicas
(22), Inglaterra (21), Canadá (6), Chile (2), Argentina (2) e Nova Zelândia (1) (Quadro 2); isto se deve à área interdisciplinar, que gerou um número de 45 artigos, à Enfermagem com 33 e a Medicina com 20 (Quadro 3). A área de Política aparece com 14, a de Psicologia com 8, a de Bioética com 5 e as de Fisioterapia e Serviço Social com apenas 1 cada. A maior parte desses artigos (80) está relacionada à subárea de Saúde do Adulto e Idoso; para a subárea de diretrizes/política temos 19, para a subárea de treinamento 11 , saúde da criança 7 , saúde coletiva 6 , saúde mental 3 e somente 1 para saúde da Mulher (Quadro 4).

Quadro 2 - Identificação dos países responsáveis pelas publicações ( $n=127)$

\begin{tabular}{|lccc|}
\hline País & $\begin{array}{c}\text { Comunicação } \\
\text { e CP }\end{array}$ & $\begin{array}{c}\text { Comunicação, } \\
\text { CP e Familia }\end{array}$ & Total \\
Estados Unidos & 73 & 14 & 87 \\
Brasil & 22 & 11 & 33 \\
Inglaterra & 21 & 4 & 25 \\
Canadá & 6 & 4 & 10 \\
Argentina & 2 & 1 & 3 \\
Chile & 2 & 0 & 2 \\
Nova Zelândia & 1 & 0 & 1 \\
Total & 127 & 34 & 161 \\
\hline
\end{tabular}

Quadro 3 - Identificação das áreas que abordam o tema $(\mathrm{n}=127)$

\begin{tabular}{|lccc|}
\hline Área & $\begin{array}{c}\text { Comunicação } \\
\text { e CP }\end{array}$ & $\begin{array}{c}\text { Comunicação, } \\
\text { CP e Familia }\end{array}$ & Total \\
Interdisciplinar & 45 & 9 & 54 \\
Enfermagem & 33 & 9 & 42 \\
Medicina & 20 & 3 & 23 \\
Política & 14 & 3 & 17 \\
Psicologia & 8 & 6 & 14 \\
Bioética & 5 & 3 & 8 \\
Serviço Social & 1 & 1 & 2 \\
Fisioterapia & 1 & 0 & 1 \\
Total & 127 & 34 & 161 \\
\hline
\end{tabular}

Quadro 4 - Identificação das subáreas que abordam o tema $(\mathrm{n}=127)$ 


\begin{tabular}{|lccc|}
\hline Subárea & $\begin{array}{c}\text { Comunicação } \\
\text { e CP }\end{array}$ & $\begin{array}{c}\text { Comunicação, } \\
\text { CP e Familia }\end{array}$ & Total \\
Adulto e Idoso & 80 & 25 & 105 \\
Diretriz* & 19 & 3 & 22 \\
Ensino & 11 & 2 & 13 \\
Coletiva & 6 & 3 & 9 \\
Criança & 7 & 1 & 8 \\
Mental & 3 & 0 & 3 \\
Mulher & 1 & 0 & 1 \\
Total & 127 & 34 & 161 \\
\hline
\end{tabular}

*Projetos/princípios para currículos pedagógicos

A atenção voltada para este assunto teve como auge a década dos anos 2000, pois do total de 127 artigos, apenas 13 são da década de 90; o restante (114) foi escrito na década seguinte. Com os descritores Comunicação, CP e Família foi encontrado apenas 1 artigo publicado na década de 90 .

O método mais utilizado nos artigos de pesquisa selecionados foi do tipo qualitativo, com um número de 107 , seguido do tipo quantitativo, com 13 no total e quanti-qualitativo, com 7 artigos (Quadro 5).

Quadro 5 - Classificação dos estudos conforme a metodologia $(\mathrm{n}=127)$

\begin{tabular}{|lccc|}
\hline Método & $\begin{array}{c}\text { Comunicação } \\
\text { e CP }\end{array}$ & $\begin{array}{c}\text { Comunicação, } \\
\text { CP e Familia }\end{array}$ & Total \\
Qualitativo & 107 & 28 & 135 \\
Quantitativo & 13 & 5 & 18 \\
Quanti-Qualitativo & 7 & 1 & 8 \\
Total & 127 & 34 & 161 \\
\hline
\end{tabular}

\section{DISCUSSÃO DOS DADOS}

Ao analisarmos o Quadro 1, referente à identificação das bases de dados com abordagem do tema, observamos que a MedLine é a base de dados que mais abarca os artigos; isto se deve, provavelmente, ao fato de tal base abranger artigos que cruzam a barreira do continente americano, enquanto nas demais bases são indexados artigos produzidos por países latino-americanos.

Em relação ao Quadro 2, identificação dos países responsáveis pelas publicações, considerando Comunicação e CP como descritores, os Estados Unidos aparecem com um grande número de artigos publicados (73). Esperávamos que isso fosse acontecer devido ao forte investimento em pesquisas e ao número elevado de revistas científicas que o país possui. O que nos surpreendeu foi o fato de o Brasil e Inglaterra possuírem 22 e 21 publicações respectivamente, pois considerando que a Inglaterra foi o "berço" dos Cuidados Paliativos, esperávamos que o número de publicações fosse maior; por outro lado, em um país que estuda e trabalha o assunto há pouco mais de uma década, a quantidade de publicações denota a importância dada por pesquisadores brasileiros ao assunto.

Quando selecionamos o descritor família à busca, os Estados Unidos e o Brasil têm praticamente o mesmo número bruto de artigos, porém se analisarmos mais a fundo, nosso país destinou 50\% das produções focando família, enquanto os Estados Unidos apenas 19,1\%. Entendemos que este resultado pode ser fruto de uma relação cultural que os brasileiros têm com suas famílias e pode ter sido influenciado pela implantação da Estratégia de Saúde da Família; não é por acaso que somos conhecidos mundialmente como um país de tradição de laços familiares fortes, isso justificaria a inserção da família em metade dos estudos brasileiros.

A identificação das áreas que abordam o tema, representada pelo Quadro 3, nos mostra que os trabalhos de Comunicação e CP têm um maior número de produções representado pela área interdisciplinar. Isto é positivo, pois entendemos que os Cuidados Paliativos são formados a partir da união das áreas da saúde, pois cada uma contribui com os seus conhecimentos específicos, e esta soma torna o cuidado ao paciente em fase terminal mais completo e eficaz. A Enfermagem é a segunda maior responsável por estudos do tema, podendo ser vista como resultado de suas próprias raízes e perfil acadêmico: o cuidado integral ao paciente. Para esta profissão, mais importante que a patologia propriamente dita, é o cuidado à pessoa acometida pela enfermidade. Ainda em relação à Enfermagem, constatamos que esta, juntamente com a interdisciplinar, contribui com o maior número de artigos focando família (9), o que era esperado, pois o cuidado a esta dimensão também é defendida fortemente pela profissão.

Analisando o Quadro 4, referente à subárea com publicações do tema, em relação à parte que consiste estudos de Comunicação e $C P$, podemos destacar a categoria adulto e idoso, com um total de 80 artigos. Isso pode ser explicado devido ao fato de que pessoas nesta faixa etária estão mais sujeitas a 
adoecer e morrer. Além disso, os Cuidados Paliativos ainda encontram-se fortemente vinculados à oncologia e, portanto, o público alvo dos estudos acaba se concentrando em adultos e idosos com problemas oncológicos. Outros campos de estudos estão encontrando seu espaço dentro dos CP, mas a sua produção científica, como saúde coletiva e criança, com seis artigos cada um, ainda é pequena.

Os resultados para a relação da categoria subárea com os descritores Comunicação, CP e Família, apesar de apresentarem números diferentes, também apontam uma concentração dos estudos na subárea de adulto e idoso, podendo ser explicada pelo mesmo motivo descrito anteriormente. Ainda sobre a Quadro 4, a subárea diretriz surge com um número de publicações importante se comparada às demais categorias. Essa subárea refere-se a princípios, projetos de currículos e políticos que buscam orientar e, até certo ponto, regulamentar o estudo, o ensino e a prática dos $\mathrm{CP}$, denotando uma preocupação constante das organizações (hospitalares, governamentais ou de ensino) com essa questão.

Ao analisarmos o número de artigos por descritores, produzidos por década, para os descritores Comunicação e $C P$ percebemos que praticamente toda a produção bibliográfica (114) levantada nesse estudo ocorreu na década de 2000, demonstrando que os Cuidados Paliativos vêm despertando um interesse cada vez maior entre estudiosos, e com isso houve um grande aumento de publicações sobre o tema se comparada à década de 90, quando foram encontradas apenas 13 publicações. Um fato que nos impressionou foi que ao considerarmos os descritores Comunicação, CP e Família encontramos apenas 1 artigo publicado na década de 90. Como já descrito anteriormente, a assistência à família configura-se como parte fundamental e inseparável dos princípios dos Cuidados Paliativos, e o fato desse aspecto estar sendo estudado com mais ênfase na década de 2000 (33) indica um maior conhecimento e amadurecimento das diretrizes e princípios dos Cuidados Paliativos por parte dos pesquisadores e profissionais que atuam nessa área.

Em relação ao Quadro 5, que traz dados sobre a classificação dos estudos conforme a metodologia, o mais utilizado pelos pesquisadores foi o método qualitativo: 107 em Comunicação e $C P$ e 28 em Comunicação, CP e Família. O método qualitativo provavelmente tem sido o mais utilizado por pesquisadores, devido à possibilidade que ele traz de analisar o tema em contextos específicos, expondo discursos de familiares, pacientes e profissionais quanto às suas vivências dessa fase de vida.

Com a elaboração deste estudo, percebemos que as publicações abordam aspectos da comunicação/ colaboração entre os hospices, a comunicação entre paciente, família e profissionais de saúde, o controle da dor e outros sintomas e as intervenções para o final da vida. Esses temas são os que aparecem com maior frequência como objeto de estudos das pesquisas que utilizam esses descritores.

Porém, também foi possível notar que existem questões emergentes que aos poucos vêm ganhando espaço nas pesquisas sobre CP. Como exemplo, podemos citar o tema Espiritualidade, que apesar de ser um dos pontos importantes nos Cuidados Paliativos, até sua definição e concepção, vem sendo pouco abordada nas pesquisas, pois apesar de muitos artigos incluírem essa questão em suas conclusões são poucos os que o tomam como objeto principal de estudo. Encontramos apenas 4 artigos que abordaram diretamente essa questão, sendo 2 na área de Enfermagem, 1 na área de Medicina e 1 na área Interdisciplinar. Todos esses artigos salientaram a necessidade de os profissionais se prepararem melhor para abordar esse tema com seus pacientes ${ }^{(10-13)}$.

Como foi dito anteriormente, as publicações científicas em CP ganharam força somente a partir da década de 2000, mas já podemos observar a elaboração de currículos específicos para cuidados paliativos, para residentes de medicina, voltado para cirurgias paliativas ${ }^{(14)}$.

Um ponto importante e satisfatório foi a constatação de que alguns estudos já se dedicam a analisar a assistência em CP a determinados grupos étnicos, seja no contexto de hospice ou no contexto de homecare, considerando suas particularidades culturais ${ }^{(11,16)}$. Encontramos também um artigo que analisou o significado de ter uma boa morte sob a perspectiva de pacientes chineses ${ }^{(16)}$. Isso evidencia que as diferenças culturais entre determinados grupos étnicos vêm se constituindo em desafios para a assistência em CP e merecem ser estudadas mais profundamente, pois os aspectos sociais e culturais são parte integrante da assistência em Cuidados Paliativos $^{(4)}$

A maior parte dos artigos traz a questão do alívio dos sintomas e da dor como aspecto principal para a melhora da qualidade de vida. Fica evidente a preocupação dos profissionais neste sentido ${ }^{(12,17)}$.

A questão da família, até nos artigos selecionados 
com o descritor, ainda é pouco abordada. Ela é tida, na maior parte dos artigos analisados, como apenas um ponto do estudo, mas não como foco. Os estudos que alavancam a discussão trazem a comunicação como aliada no atendimento do paciente e sua família, ou que a escuta é importante para auxiliar no enfrentamento e possibilitar o acolhimento do paciente e sua família. Observamos que a família vem somada ao paciente, não é objeto de estudo propriamente dito ${ }^{(18,19)}$. Isso é importante, pois sua soma é uma unidade do cuidado, mas cremos que a família também pode e deve receber um atendimento qualificado e diferenciado para suprir suas expectativas, afinal, a visão de mundo de quem está morrendo é diferente da visão daqueles que passarão pela experiência de morte de alguém, mas que continuarão vivendo; por isso, um avanço nas pesquisas destinadas ao estudo de familiares de pacientes em fase terminal faz-se relevante.

\section{CONSIDERAÇÕES FINAIS}

Inegavelmente, na última década, os $\mathrm{CP}$ vêm ganhando espaço tanto nos centros de estudos quanto na prática e ensino em saúde. Mas ainda é preciso avançar em alguns aspectos específicos para que a assistência em Cuidados Paliativos se torne cada vez mais individualizada, possibilitando uma abordagem que vá além dos aspectos biológicos e inclua os aspectos sociais, espirituais e emocionais dos pacientes submetidos a essa terapêutica, proporcionando assim uma melhor qualidade de vida e a redução do sofrimento tanto do paciente quanto de sua família ${ }^{(20)}$.

Apesar desse avanço, é notória a necessidade de um maior comprometimento das organizações políticas e administrativas com o ensino, a prática e a pesquisa sobre Cuidados Paliativos, pois o baixo número de publicações para a área de Política pode significar que essas organizações não estão garantindo os recursos financeiros, materiais e humanos de forma a programar estratégias e garantir a manutenção em padrões satisfatórios dos serviços especializados no cuidado aos pacientes em fase terminal.

Com relação à família, o número de publicações que a considera como foco é muito pequeno quando comparado com outro tipo de estudo (Comunicação e Cuidados Paliativos). Fica evidente que esta instituição familiar sofre uma intensa transformação, às vezes nunca sentida por nenhum membro antes, provocando dúvidas, medos e confusões, que seriam mais facilmente resolvidas se esta for tomada como premissa assistencial dos Cuidados Paliativos.

Por meio deste estudo percebemos que a forma mais fácil e eficaz de entendimento da família e sua aproximação para a assistência paliativa é através da comunicação, pois este instrumento do Cuidado consegue "tocar" e confortar muitas vezes mais do que qualquer outro medicamento ${ }^{(21)}$.

A conversa entre o profissional de saúde e a família pode desvendar muitos anseios, medos e dúvidas presentes nesta dimensão e, assim, proporcionar a criação de vínculo, importante e necessário nesta etapa da vida. Para que este vínculo seja efetivo é necessário que os profissionais sejam treinados em comunicação terapêutica e também desmitificar a ideia de que a família "só atrapalha” ou que o cuidado é somente para o paciente. Seria importante se isto acontecesse desde a graduação, para que o entendimento sobre o tema já esteja enraizado quando formados. Para isso, sugerimos estudos com alunos de graduação da área da saúde, a fim de avaliar qual o grau de conhecimento em relação ao assunto e qual a importância que estes futuros profissionais veem na inserção da família, vista como um dos focos básicos nos cuidados paliativos.

Outra sugestão seria a formulação de novas pesquisas em Cuidados Paliativos, mas que tenham como objetivo o estudo do processo de morrer sob o ponto de vista da família, pois, por meio delas, fica um entendimento mais profundo sobre como é passar por esta vivência.

\section{REFERÊNCIAS}

1. Susaki TT, Silva MJP, Possari JF. Identificação das fases do processo de morrer pelos profissionais de saúde. Acta Paul Enferm. 2006 Abr/Jun;9(2):144-9.

2. Pessini L. A filosofia dos cuidados paliativos: uma resposta diante da obstinação terapêutica. In: Pessini L, Bertachini L. Humanização e cuidados paliativos. São Paulo: Loyola. 2004. P.181-208.

3. Araújo MT, Silva MJP. Cuidados paliativos na UTI: possibilidade de humanização do processo de morrer. RSBC. 2006;40-44.

4. World Health Organization-Who. Who Definition of Palliative Care. [acesso em 2008 Mar 28] .Disponível: http://www.who.int/cancer/palliative/definition/en.

5. Ministério da Saúde (BR). Secretaria de Vigilância em Saúde. Programa Nacional de DST e aids. Rotinas de 
assistência domiciliar terapêutica (ADT) em HIV/Aids. Brasília; 2007.

6. Leahey M, Wright LM. Enfermeiras e famílias: um guia para avaliação e intervenção na família. $3^{\mathrm{a}}$ ed. São Paulo: Roca; 2002.

7. Fournier JP. In her eyes. Carin. 2002 Oct;21(10):22-3.

8. Hallenbeck JL. Communication across cultures. J Palliat Med. 2004 Jun;7(3):477-80.

9. McGoldrick L. Specialist palliative care nursing in the community. Nurs Times. 1999Ago;95(31):53.

10. Haag BA, Sellers SC. Spiritual nursing interventions. J Holist Nurs 1998 Set;16(3):338-54.

11. Clarke H, McGrath P. Creating the space for spiritual talk: insights from survivors of hematological malignancies. Aust Health Rev. 2003;26(3):116-32.

12. Bowen DJ, Deyo R, Hart A, Kohlwes RJ, Rhodes R. Hospice patients' attitudes regarding spiritual discussions with their doctors. Am J Hosp Palliat Care. 20003 Mar/Abr;20(2):135-9.

13. Rodrigues IG, Zago MMF. Enfermagem em Cuidados Paliativos. Mundo da Saúde. 2003 Jan/Mar;27(1):8992.

14. Harrington DT, Klaristenfeld DD, Miner TJ. Teaching palliative care and end-of-life issues: a core curriculum for surgical residents. Ann Surg Oncol; 2007 Jun;14(6):1801-6.

15. Beutter MB, DavidHizar R. A home care provider's challenge-caring for the Hispanic client at home. J Pract Nurs 1999 St;49(3):26-33.

16. Mak MH. Awareness of gying an experience of Chinese patients with terminal cancer. Omega (Westport). 2001;43(3):259-79.

17. Miceli AVP. Dor crônica e subjetividade em oncologia. Rev Bras Oncol. 2002 Jul/Set;48(3):363-73.

18. Esslinger, I. O paciente, a equipe de saúde e o cuidador: de quem é a vida afinal? Um estudo acerca do morrer com dignidade. Mundo da Saúde. 2001 Jul/ Set;27(3):373-82.

19. Silva, MJP. Comunicação com pacientes fora de possibilidades terapêuticas: reflexões. Mundo da Saúde. 2003 Jan/Mar;27(1):64-70.
20. Girond, JBR; Waterkemper, R. Sedação, eutanásia e o processo de morrer do paciente com câncer em cuidados paliativos: compreendendo conceitos e inter-relações. Cogitare Enferm. 2006 Set/Dez;11(3):258-63.

21. Araújo MT, Silva MJP. Quando "uma palavra de carinho conforta mais que um medicamento": necessidades e expectativas de pacientes sob cuidados paliativos [dissertação]. São Paulo (SP): Escola de Enfermagem, Universidade de São Paulo; 2006. 